\title{
Solvable monoids with commuting idempotents
}

\author{
Manuel Delgado ${ }^{1}$ and Vítor H. Fernandes ${ }^{2}$
}

AMS Mathematics Subject Classification: 20M07, 20M18, 20F14, 20F19, 20M35, 68Q70

\begin{abstract}
The notion of Abelian kernel of a finite monoid extends the notion of derived subgroup of a finite group. In this line, an extension of the notion of solvable group to monoids is quite natural: they are the monoids such that the chain of Abelian kernels ends with the submonoid generated by the idempotents. We prove in this paper that the finite idempotent commuting monoids satisfying this property are precisely those whose subgroups are solvable.
\end{abstract}

\section{Introduction}

The computability of the kernel $\mathrm{K}_{\mathrm{H}}(M)$ of a finite monoid $M$ relative to a pseudovariety $\mathrm{H}$ of groups is closely related to the decidability of Mal'cev products where the second factor is a pseudovariety of groups. In fact, for a decidable pseudovariety $\mathrm{V}$ of monoids and a pseudovariety $\mathrm{H}$ of groups, being able to compute $\mathrm{K}_{\mathrm{H}}(M)$, for any finite monoid $M$, automatically guarantees the decidability of the pseudovariety of monoids

$$
\mathrm{V}\left(\mathrm{m} \mathrm{H}=\left\{M \text { finite monoid } \mid \mathrm{K}_{\mathrm{H}}(M) \in \mathrm{V}\right\}\right.
$$

which is well-known to be the Mal'cev product of $\mathrm{V}$ and $\mathrm{H}$. As the Mal'cev product of pseudovarieties of monoids interests many researchers, the importance of computing kernels is out of question. The popularity of the kernel notion comes from a conjecture of J. Rhodes that proposed an algorithm to perform the computation of the kernel relative to the pseudovariety of all finite groups. It is known as the Rhodes Type II Conjecture and survived as a conjecture almost 20 years. It became a theorem after independent and deep work of Ash [7] and Ribes and Zalesskiı [22]. The history and some consequences of the Type II Conjecture may be found in [15]. The results of Ash and of Ribes and Zalesskiu that led to its proof have since then been extended in various directions $[6,16,3,4]$ and several connections between both results have also been found [11, 16, 3, 4].

The Abelian counterpart of the Rhodes Type II Conjecture was solved by the first author in [9] and the algorithm there obtained to compute the Abelian kernel of a finite

\footnotetext{
${ }^{1}$ The author gratefully acknowledges support of FCT through the Centro de Matemática da Universidade do Porto and the FCT and POCTI Project POCTI/32817/MAT/2000 which is funded in cooperation with the European Community Fund FEDER and of project INTAS 99-1224.

${ }^{2}$ The author gratefully acknowledges support of FCT and FEDER, within the project "Álgebra e Aplicações", POCTI/32440/MAT/1999, of Centro de Álgebra da Universidade de Lisboa.
} 
monoid was then optimised and rendered usable in practice in [10]. The monoids used to test an implementation of this algorithm in GAP [27] belong to some classes of (inverse) monoids which had been object of study by the second author. For instance in [14] several structural properties of the monoids $\mathcal{P O \mathcal { I } _ { n }}$ of all injective order preserving partial transformations on a chain with $n$ elements were studied and a presentation for these monoids was given. Inspired by the results obtained through lots of practical calculations and using this presentation, we computed in [12] the Abelian kernel of the monoids $\mathcal{P} \mathcal{O I}_{n}$. Analogous results were also obtained in the same paper for the monoids $\mathcal{P} \mathcal{O P} \mathcal{I}_{n}$ of all injective orientation preserving partial transformations on a chain with $n$ elements. A natural question then arose: what happens when we compute the Abelian kernel of the Abelian kernel and so on? This was a strong motivation for the present paper since, as the Abelian kernel of a finite group $G$ coincides with the derived subgroup of $G$, we can use iterations of the Abelian kernel to define solvable monoids, just as we use iterations of the derived group to define solvable groups. Let us observe that there are other properties of groups, for example, being poly-cyclic, which have attracted the attention of many researchers and could be object of the same kind of generalisation, but we will not explore these in the present paper.

Although the generalisation to monoids of the important notion of solvable group is sufficient to motivate all the results in this paper, our motivation goes beyond this. This follows from the various questions raised in Section 4, but will also be briefly explained below.

Besides the Mal'cev product, another operator that has attracted the attention of semigroup theorists is the semidirect product. The semidirect product $\mathrm{V} * \mathrm{~W}$ of the pseudovarieties of monoids $\mathrm{V}$ and $\mathrm{W}$ is the pseudovariety generated by all monoidal semidirect products $M * N$, where $M \in \mathrm{V}$ and $N \in \mathrm{W}$. The underlying set of a monoidal semidirect product $M * N$ is the direct product $M \times N$ of the underlying sets of the monoids $M$ and $N$. The direct product of two monoids is an example of a semidirect product. For more details on semidirect products (either the semigroup or the monoidal version) we refer the reader to [1]. There is an easy connection between both operators: $\mathrm{V} * \mathrm{H} \subseteq \mathrm{V} m \mathrm{H}$, for all pseudovarieties $\mathrm{V}$ of monoids and $\mathrm{H}$ of groups. The equality holds in some important cases, but not always, as we discuss in Section 4.

A problem that has interested many semigroup theorists for the past few decades is the decidability of the semidirect product of pseudovarieties in general and the decidability of iterated semidirect products, possibly for particular pseudovarieties. Notice that a positive answer for the question of the decidability of iterated semidirect products whose factors are the classes of all finite groups and of all finite aperiodic semigroups would solve the problem of the "decidability of the complexity", which is undoubtedly the most famous problem in finite semigroup theory.

Just as computing kernels is related to the decidability of Mal'cev products, computing iterated kernels is related to the decidability of iterated Mal'cev products. As Mal'cev products and semidirect products coincide in some important cases, this paper is also related to the important problem of the decidability of iterated semidirect products.

The first section of the present paper is mostly devoted to background.

In the second section we state some results that hold for any pseudovariety $\mathrm{H}$ of groups. The definition of $\mathrm{H}$-solvability is given in this section. Some of the former general results are used in the third section where we consider specifically the pseudovariety of Abelian 
groups and give a characterisation of Ab-solvable monoids with commuting idempotents: they are precisely those whose subgroups are solvable.

The last section contains comments and questions.

After the submission of the present paper, the authors together with Margolis and Steinberg, found a generalisation of the main result of the present paper (Theorem 3.6). These new results will also appear in the International Journal of Algebra and Computation.

\section{Definitions and background}

For basic background on Green relations and inverse semigroups, see Howie's book [18]. For basic notions related with rational languages we refer the reader to Pin's book [20]. For background on profinite topologies, see [2].

Let $M$ be a monoid. Recall the definition of the quasi-order $\leq_{\mathcal{y}}$ associated to the Green relation $\mathcal{J}$. For all $u, v \in M$,

$$
u \leq_{\mathfrak{g}} v \text { if and only if } M u M \subseteq M v M
$$

Notice that, for every $u, v \in M, u \mathcal{J} v$ if and only if $u \leq_{\mathcal{J}} v$ and $v \leq_{\mathcal{J}} u$. Denote by $J_{u}$ the J-class of the element $u \in M$. As usual, a partial order relation $\leq_{\mathcal{g}}$ is defined on the set $M / \mathcal{J}$ by setting, for all $u, v \in M, J_{u} \leq_{\mathcal{J}} J_{v}$ if and only if $u \leq_{\mathcal{J}} v$. Given $u, v \in M$, we write $u<_{\mathfrak{g}} v$ and $J_{u}<_{\mathfrak{J}} J_{v}$ if and only if $u \leq_{\mathfrak{J}} v$ and $(u, v) \notin \mathcal{J}$.

Similar notations are used for the other Green relations $\mathcal{R}, \mathcal{L}, \mathcal{H}=\mathcal{R} \cap \mathcal{L}$ and $\mathcal{D}=\mathcal{R} \circ \mathcal{L}$. Notice that for a finite monoid $\mathcal{J}=\mathcal{D}$.

Let $X$ be a subset of $M$. We denote by $\langle X\rangle$ the submonoid of $M$ generated by $X$ and by $E(M)$ the set of the idempotents of $M$.

A relational morphism of semigroups $\tau: S \nrightarrow T$ is a function from $S$ into the power set of $T$, such that:

(a) For all $s \in S, \tau(s) \neq \emptyset$;

(b) For all $s_{1}, s_{2} \in S, \tau\left(s_{1}\right) \tau\left(s_{2}\right) \subseteq \tau\left(s_{1} s_{2}\right)$.

When $S$ and $T$ are monoids, $\tau$ is a relational morphism of monoids if it satisfies (a), (b) and

(c) $1 \in \tau(1)$.

So, a relational morphism $\tau: S \multimap T$ is, in particular, a relation in $S \times T$ and we may compose relational morphisms in the obvious way. Homomorphisms, seen as relations, and inverses of onto homomorphisms are examples of relational morphisms.

From now on $\mathrm{H}$ always denotes a pseudovariety of groups.

The $\mathrm{H}$-kernel (or kernel relative to $\mathrm{H}$ ) of a finite semigroup $S$ is the subsemigroup $\mathrm{K}_{\mathrm{H}}(S)=\bigcap \tau^{-1}(1)$, with the intersection being taken over all groups $G \in \mathrm{H}$ and all relational morphisms of semigroups $\tau: S \multimap G$. The $\mathrm{H}$-kernel of a finite monoid is defined analogously, using relational morphisms of monoids instead of relational morphisms of 
semigroups. Computing the $\mathrm{H}$-kernel of a finite semigroup can be reduced to the computation of the $\mathrm{H}$-kernel of a finite monoid. In fact, it is straightforward that, for a finite semigroup $S, \mathrm{~K}_{\mathrm{H}}(S)=\mathrm{K}_{\mathrm{H}}\left(S^{1}\right) \cap S$, where $S^{1}$ denotes the monoid obtained from $S$ by adding an identity, if $S$ has none, or $S$ itself, otherwise. Thus, when one wants to deal with $\mathrm{H}$-kernels, as is the case in this paper, it is sufficient to treat the monoid case.

Next we collect some results related to the Ab-kernel of a finite monoid that were first stated in [9] and will be used in Section 3. The Ab-kernel is usually referred as the Abelian kernel.

The $n$-generated free Abelian group $\mathbb{Z}^{n}$ is considered endowed with the profinite group topology, which is the least topology rendering continuous all homomorphisms into finite groups. The topological closure of a subset $X$ of a topological space will be denoted by $\bar{X}$.

Proposition 1.1 For $a, b_{1}, \ldots, b_{r} \in \mathbb{N}^{n}$, the topological closure of the subset $a+b_{1} \mathbb{N}+$ $\cdots+b_{r} \mathbb{N}$ of $\mathbb{Z}^{n}$ is $a+b_{1} \mathbb{Z}+\cdots+b_{r} \mathbb{Z}$, i.e. $\overline{a+b_{1} \mathbb{N}+\cdots+b_{r} \mathbb{N}}=a+b_{1} \mathbb{Z}+\cdots+b_{r} \mathbb{Z}$.

In order to make our notation more comprehensive, we will use use subscripts in certain components of the elements of $\mathbb{Z}^{n}$. For instance, we write $\left(0, \ldots, 0,1_{(i)}, 0, \ldots, 0\right)$ with the meaning of " $(0, \ldots, 0,1,0, \ldots, 0)$ ( 1 is in the position $i)$ ".

Let $A=\left\{a_{1}, \ldots, a_{n}\right\}$. The canonical homomorphism $\gamma: A^{*} \rightarrow \mathbb{Z}^{n}$ given by

$$
\gamma\left(a_{i}\right)=\left(0, \ldots, 0,1_{(i)}, 0, \ldots, 0\right),
$$

for $1 \leq i \leq n$, will be widely used. In [9] an algorithm is given to compute the image under $\gamma$ of a rational language of $A^{*}$ given by a rational expression.

Suppose that $M$ is an $A$-generated finite monoid and let $\varphi: A^{*} \rightarrow M$ be an onto homomorphism. Then we have the following proposition, which gives an algorithm to compute the Abelian kernel of a finite monoid.

Proposition 1.2 An element $x \in M$ is in the Abelian kernel of $M$ if and only if $(0, \ldots, 0) \in \overline{\gamma\left(\varphi^{-1}(x)\right)}$.

Since a finite group may be seen as a finite monoid, the notion of $\mathrm{H}$-kernel may also be applied to groups. The following holds (see [9]):

Proposition 1.3 The $\mathrm{H}$-kernel of a finite group $G$ is the smallest normal subgroup $H$ of $G$ such that the quotient $G / H$ belongs to $\mathrm{H}$.

The derived subgroup of a group $G$ is the subgroup $G^{\prime}$ generated by the commutators $x y x^{-1} y^{-1}(x, y \in G)$. Observing that $G^{\prime}$ is the smallest normal subgroup $H$ of $G$ such that the quotient $G / H$ is Abelian, one immediately obtains the following corollary. Nevertheless, we include a proof of this result to illustrate our techniques.

Corollary 1.4 The Abelian kernel of a finite group $G$ is precisely its derived subgroup $G^{\prime}$. 
Proof. For the canonical projection $p: G \rightarrow G / G^{\prime}$ we have $p^{-1}(1)=G^{\prime}$, it follows that $\mathrm{K}_{\mathrm{Ab}}(G) \subseteq G^{\prime}$, since $p$ is a relational morphism into a finite Abelian group.

In order to prove the reverse inclusion, let us consider a commutator $c=x y x^{-1} y^{-1}$ of $G$. We have to prove that $c \in \mathrm{K}_{\mathrm{Ab}}(G)$.

Suppose now that $G$ is $B$-generated (as a group or as a semigroup: recall that $G$ is finite), where $B=\left\{b_{1}, \ldots, b_{n}\right\}$ is a finite set with $n$ elements. Let $\psi: B^{*} \rightarrow G$ be a surjective homomorphism. Let $A=B \cup B^{-1}$, where $B^{-1}=\left\{b^{-1}: b \in B\right\}$ is a disjoint copy of $B$. Now extend $\psi$ to a homomorphism $\varphi: A^{*} \rightarrow G$ by defining $\varphi\left(b^{-1}\right)=(\psi(b))^{-1}$, for $b \in B$. Consider now the group $G$ together with the homomorphism $\varphi$ as an $A$-generated monoid. We fix the ordering $b_{1}, \ldots, b_{n}, b_{1}^{-1}, \ldots, b_{n}^{-1}$ for the elements of $A$ and consider the canonical homomorphism $\gamma: A^{*} \rightarrow \mathbb{Z}^{2 n}$ as above.

Let $u=x_{1} \cdots x_{r} \in A^{*}\left(x_{1}, \ldots, x_{r} \in A\right)$ be such that $\varphi(u)=x$ and let $v=y_{1} \cdots y_{s} \in$ $A^{*}\left(y_{1}, \ldots, y_{s} \in A\right)$ be such that $\varphi(v)=y$. Then $w=x_{1} \cdots x_{r} y_{1} \cdots y_{r} x_{r}^{-1} \cdots x_{1}^{-1} y_{s}^{-1} \cdots y_{1}^{-1}$ is such that $\varphi(w)=c$.

As inserting in $w$ a factor consisting of a letter and its (formal) inverse we obtain a word $w^{\prime}$ such that $\varphi\left(w^{\prime}\right)=\varphi(w)$, by Proposition 1.1, we have that $\overline{\gamma\left(\varphi^{-1}(c)\right)}$ contains the set

$$
\gamma(w)+\left(1_{(1)}, 0, \ldots, 0,1_{(n+1)}, 0, \ldots, 0\right) \mathbb{Z}+\cdots+\left(0, \ldots, 0,1_{(n)}, 0, \ldots, 0,1_{(2 n)}\right) \mathbb{Z} .
$$

Since $|w|_{a}=|w|_{a^{-1}}$ for each letter $a$, this is equal to the set

$$
\left(1_{(1)}, 0, \ldots, 0,1_{(n+1)}, 0, \ldots, 0\right) \mathbb{Z}+\cdots+\left(0, \ldots, 0,1_{(n)}, 0, \ldots, 0,1_{(2 n)}\right) \mathbb{Z} .
$$

Thus $(0, \ldots, 0) \in \overline{\gamma\left(\varphi^{-1}(c)\right)}$. We conclude using Proposition 1.2.

Just as one forms the derived series $\left\{G^{(i)} \mid i \geq 0\right\}$ of a group $G$ by iterating the the derived subgroup operation, one can iterate the computation of the $\mathrm{H}$-kernel of a finite monoid $M$. We define $\mathrm{K}_{\mathrm{H}}^{(n)}(M)$ recursively as follows:

- $\mathrm{K}_{\mathrm{H}}^{(0)}(M)=M$;

- $\mathrm{K}_{\mathrm{H}}^{(n)}(M)=\mathrm{K}_{\mathrm{H}}\left(\mathrm{K}_{\mathrm{H}}^{(n-1)}(M)\right)$, for $n \geq 1$.

We also consider iterations of Mal'cev and semidirect products. Here are the formal definitions:

Let $\mathrm{V}$ be a pseudovariety of monoids. For $n \geq 1$, let us define the operator $(m)^{n}$ recursively as follows:

- $\mathrm{V} \rightarrow{ }^{1} \mathrm{H}=\mathrm{V} \rightarrow \mathrm{H}$;

- $\mathrm{V} m \mathrm{~m}^{n+1} \mathrm{H}=\left(\mathrm{V}(m)^{n} \mathrm{H}\right)(\mathrm{m} \mathrm{H}$.

An operator $*^{n}$ can be defined analogously:

- $\mathrm{V} *^{1} \mathrm{H}=\mathrm{V} * \mathrm{H}$;

- $\mathrm{V} *^{n+1} \mathrm{H}=\left(\mathrm{V} *^{n} \mathrm{H}\right) * \mathrm{H}$.

Some facts about the above operators holding for $n=1$ generalise immediately to any $n \geq 1$ :

Proposition 1.5 For all $n \geq 1$, we have $\mathrm{V} \rightarrow{ }^{n} \mathrm{H}=\left\{M\right.$ finite monoid $\left.\mid K_{\mathrm{H}}^{(n)}(M) \in \mathrm{V}\right\}$ and $\mathrm{V} *^{n} \mathrm{H} \subseteq \mathrm{V} \rightarrow{ }^{n} \mathrm{H}$. 


\section{General Results}

This section is divided into 4 subsections, the first of which is devoted to some basic results on the $\mathrm{H}$-kernel and its iterations. In the second subsection we propose the definition of $\mathrm{H}$-solvable monoid. The general results proved in that subsection in conjunction with the results of Section 3 for the case $\mathrm{H}=$ Ab show, in particular, that the monoids with commuting idempotents which are Ab-solvable according to our definition are precisely the monoids whose subgroups are solvable.

While studying semigroups, regularity plays often an important role. It is also the case here. Some results concerning regularity are proved in the third subsection. The $\mathrm{H}$-solvability of a monoid whose regular elements are idempotents is obtained as an easy consequence, and stated in the fourth subsection.

\subsection{Kernels}

We collect in a single statement all the results of this subsection. Some of them are simple observations to be used later while others require detailed proofs.

Theorem 2.1 Let $M$ and $N$ be two finite monoids and $n \geq 1$. Then:

1. $\langle E(M)\rangle \subseteq K_{\mathrm{H}}^{(n)}(M)$.

2. $K_{\mathrm{H}}^{(n)}(N) \subseteq K_{\mathrm{H}}^{(n)}(M)$, if $N$ is a submonoid of $M$.

3. $K_{\mathrm{H}}^{(n)}(\varphi(M))=\varphi\left(K_{\mathrm{H}}^{(n)}(M)\right)$, for an onto homomorphism $\varphi: M \longrightarrow N$.

4. $K_{\mathrm{H}}^{(n)}(M \times N)=K_{\mathrm{H}}^{(n)}(M) \times K_{\mathrm{H}}^{(n)}(N)$.

5. $K_{\mathrm{H}}^{(n)}(M) \times K_{\mathrm{H}}^{(n)}(N) \subseteq K_{\mathrm{H}}^{(n)}(M * N)$, with $M * N$ a monoidal semidirect product.

Proof. The statements 1 and 2 are easy and well-known for $n=1$. Using induction one obtains the results for all natural numbers.

For statement 3, take $y \in \varphi\left(\mathrm{K}_{\mathrm{H}}(M)\right)$ and let $x \in \mathrm{K}_{\mathrm{H}}(M)$ be such that $y=\varphi(x)$. Let $G \in \mathrm{H}$ and let $\tau: N \multimap G$ be a relational morphism. Then $\xi=\tau \circ \varphi: M \multimap G$

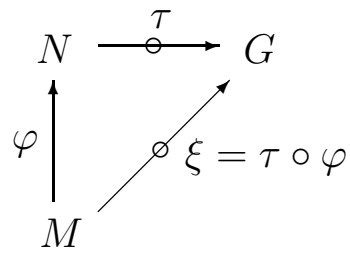

is a relational morphism and, since $x \in \mathrm{K}_{\mathrm{H}}(M)$, we have $1 \in \xi(x)=\tau(\varphi(x))=\tau(y)$, whence $y \in \tau^{-1}(1)$. Thus $y \in \mathrm{K}_{\mathrm{H}}(N)$ and so $\varphi\left(\mathrm{K}_{\mathrm{H}}(M)\right) \subseteq \mathrm{K}_{\mathrm{H}}(N)$.

Conversely, consider a group $G \in \mathrm{H}$ and a relational morphism $\tau: M \rightarrow G$ such that $\mathrm{K}_{\mathrm{H}}(M)=\tau^{-1}(1)$. Such a relational morphism exists by a finiteness argument.

Then $\xi=\tau \circ \varphi^{-1}: N \mapsto G$ 


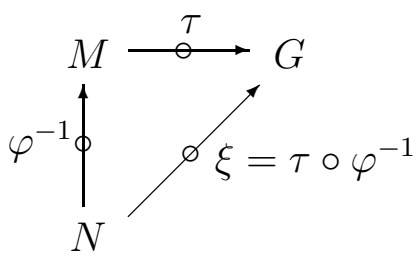

is a relational morphism since $\varphi$ is an onto homomorphism. Let $y \in \mathrm{K}_{\mathrm{H}}(N)$. Then $1 \in$ $\xi(y)=\tau\left(\varphi^{-1}(y)\right)$, whence $1 \in \tau(x)$, for some $x \in \varphi^{-1}(y)$. Hence, $x \in \tau^{-1}(1)=\mathrm{K}_{\mathrm{H}}(M)$ and $y=\varphi(x)$. Thus $y \in \varphi\left(\mathrm{K}_{\mathrm{H}}(M)\right)$ and so $\mathrm{K}_{\mathrm{H}}(N) \subseteq \varphi\left(\mathrm{K}_{\mathrm{H}}(M)\right)$.

We have thus proved that $\mathrm{K}_{\mathrm{H}}(N)=\varphi\left(\mathrm{K}_{\mathrm{H}}(M)\right)$. Therefore, it follows by induction that

$$
\mathrm{K}_{\mathrm{H}}^{(n)}(\varphi(M))=\varphi\left(\mathrm{K}_{\mathrm{H}}^{(n)}(M)\right)
$$

for all $n \in \mathbb{N}$.

To prove statements 4 and 5 , let $M * N$ be any monoidal semidirect product of $M$ and $N$. Given a subset $X$ of $M \times N$, denote by $\langle X\rangle_{*}$ the submonoid of $M * N$ generated by $X$. Let $M^{\prime}$ and $N^{\prime}$ be submonoids of $M$ and $N$, respectively. Take $a \in \mathrm{K}_{\mathrm{H}}\left(M^{\prime}\right)$ and $b \in \mathrm{K}_{\mathrm{H}}\left(N^{\prime}\right)$. Let $G \in \mathrm{H}$ and let $\tau:\left\langle M^{\prime} \times N^{\prime}\right\rangle_{*} \rightarrow G$ be a relational morphism. Consider the inclusion maps

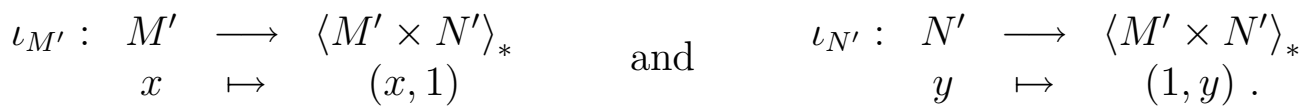

It is a routine matter to show that both $\iota_{M^{\prime}}$ and $\iota_{N^{\prime}}$ are homomorphisms. Notice that we just need the monoidal property of $M * N$ to show that $\iota_{N^{\prime}}$ is a homomorphism. Thus $\tau_{M^{\prime}}=\tau \circ \iota_{M^{\prime}}: M^{\prime} \multimap G$ and $\tau_{N^{\prime}}=\tau \circ \iota_{N^{\prime}}: N^{\prime} \multimap G$ are relational morphisms, whence $1 \in \tau_{M^{\prime}}(a)$ and $1 \in \tau_{N^{\prime}}(b)$, i.e. $1 \in \tau(a, 1)$ and $1 \in \tau(1, b)$ and so

$$
1=1 \cdot 1 \in \tau(a, 1) \tau(1, b) \subseteq \tau((a, 1)(1, b))=\tau\left(a 1^{1}, b\right)=\tau(a 1, b)=\tau(a, b),
$$

i.e. $(a, b) \in \tau^{-1}(1)$. Hence $(a, b) \in \mathrm{K}_{\mathrm{H}}\left(\left\langle M^{\prime} \times N^{\prime}\right\rangle_{*}\right)$. Thus

$$
\mathrm{K}_{\mathrm{H}}\left(M^{\prime}\right) \times \mathrm{K}_{\mathrm{H}}\left(N^{\prime}\right) \subseteq \mathrm{K}_{\mathrm{H}}\left(\left\langle M^{\prime} \times N^{\prime}\right\rangle_{*}\right) \subseteq \mathrm{K}_{\mathrm{H}}(M * N) .
$$

In particular, we have $\mathrm{K}_{\mathrm{H}}(M) \times \mathrm{K}_{\mathrm{H}}(N) \subseteq \mathrm{K}_{\mathrm{H}}(M \times N)$.

On the other hand, take $(a, b) \in \mathrm{K}_{\mathrm{H}}(M \times N)$. Let $G \in \mathrm{H}$ and let $\tau_{M}: M \rightarrow G$ be a relational morphism. Consider the projection

$$
\begin{aligned}
\pi_{M}: M \times N & \longrightarrow M \\
(x, y) & \mapsto x
\end{aligned}
$$

and the relational morphism $\tau=\tau_{M} \circ \pi_{M}: M \times N \multimap G$. Then $1 \in \tau(a, b)=\tau_{M}(a)$, i.e. $a \in \tau_{M}^{-1}(1)$. Thus $a \in \mathrm{K}_{\mathrm{H}}(M)$. Similarly, one shows that $b \in \mathrm{K}_{\mathrm{H}}(N)$ and so $\mathrm{K}_{\mathrm{H}}(M \times N) \subseteq \mathrm{K}_{\mathrm{H}}(M) \times \mathrm{K}_{\mathrm{H}}(N)$.

Therefore, we have proved that $\mathrm{K}_{\mathrm{H}}(M \times N)=\mathrm{K}_{\mathrm{H}}(M) \times \mathrm{K}_{\mathrm{H}}(N)$. By induction, we also obtain

$$
\mathrm{K}_{\mathrm{H}}^{(n)}(M \times N)=\mathrm{K}_{\mathrm{H}}^{(n)}(M) \times \mathrm{K}_{\mathrm{H}}^{(n)}(N),
$$

for all $n \in \mathbb{N}$. This completes the proof of statement 4 . 
The inclusion $\mathrm{K}_{\mathrm{H}}^{(n)}(M) \times \mathrm{K}_{\mathrm{H}}^{(n)}(N) \subseteq \mathrm{K}_{\mathrm{H}}^{(n)}(M * N)$, holds for $n=1$ as follows from (1). To prove that it holds for $n \in \mathbb{N}$ we use induction. Suppose that $\mathrm{K}_{\mathrm{H}}^{(n)}(M) \times \mathrm{K}_{\mathrm{H}}^{(n)}(N) \subseteq$ $\mathrm{K}_{\mathrm{H}}^{(n)}(M * N)$. Hence $\left\langle\mathrm{K}_{\mathrm{H}}^{(n)}(M) \times \mathrm{K}_{\mathrm{H}}^{(n)}(N)\right\rangle_{*} \subseteq \mathrm{K}_{\mathrm{H}}^{(n)}(M * N)$. Then, we have

$$
\begin{aligned}
\mathrm{K}_{\mathrm{H}}^{(n+1)}(M) \times \mathrm{K}_{\mathrm{H}}^{(n+1)}(N) & =\mathrm{K}_{\mathrm{H}}\left(\mathrm{K}_{\mathrm{H}}^{(n)}(M)\right) \times \mathrm{K}_{\mathrm{H}}\left(\mathrm{K}_{\mathrm{H}}^{(n)}(N)\right) \\
& \subseteq \mathrm{K}_{\mathrm{H}}\left(\left\langle\mathrm{K}_{\mathrm{H}}^{(n)}(M) \times \mathrm{K}_{\mathrm{H}}^{(n)}(N)\right\rangle_{*}\right) \\
& \subseteq \mathrm{K}_{\mathrm{H}}\left(\mathrm{K}_{\mathrm{H}}^{(n)}(M * N)\right)=\mathrm{K}_{\mathrm{H}}^{(n+1)}(M * N),
\end{aligned}
$$

as required.

\subsection{Solvability}

For a group theoretical property $P$, a group $G$ is said to be a poly- $P$ group if there is a chain

$$
G=G_{0} \geq G_{1} \geq \cdots \geq G_{r}=1
$$

such that, for $i=1, \ldots, r, G_{i}$ is a normal subgroup of $G_{i-1}$ and the quotient $G_{i-1} / G_{i}$ satisfies the property $P$. Properties largely considered in the literature $[23,26]$ are those of being commutative and of being cyclic. The corresponding poly- $P$ groups are known as solvable groups and poly-cyclic groups respectively.

A group $G$ is easily seen to be solvable if and only if there exists a positive integer $n$ such that $G^{(n)}=\{1\}$.

Let $P_{\mathrm{H}}$ be the property of being an element of $\mathrm{H}$. As in the solvable case, in view of Proposition 1.3, it is easy to see that a finite group $G$ is a poly- $P_{\mathrm{H}}$ group if and only if there exists a positive integer $n$ such that $\mathrm{K}_{\mathrm{H}}^{(n)}(G)=\{1\}$.

Rather than using the terminology poly- $P_{\mathrm{H}}$, one could use the apparently more natural: $\mathrm{H}$-solvable. This is what we will do in the monoid case.

We say that a finite monoid $M$ is $\mathrm{H}$-solvable if $\mathrm{K}_{\mathrm{H}}^{(n)}(M)=\langle E(M)\rangle$, for some nonnegative integer $n$.

Let $\mathrm{V}$ be a pseudovariety of monoids. Define the class

$$
\mathrm{V}_{\mathrm{Hsol}}=\{M \in \mathrm{V} \mid M \text { is } \mathrm{H} \text {-solvable }\} \text {. }
$$

For $\mathrm{H}=\mathrm{Ab}$, we denote $\mathrm{V}_{\mathrm{Hsol}}$ simply by $\mathrm{V}_{\text {sol }}$.

We say that a pseudovariety $\mathrm{V}$ of monoids is $\mathrm{H}$-solvable if all its elements are $\mathrm{H}$-solvable monoids, i.e. if $\mathrm{V}=\mathrm{V}_{\mathrm{Hsol}}$.

As examples of $\mathrm{H}$-solvable pseudovarieties of monoids, we immediately have the pseudovariety B of all finite bands, as well as each of its subpseudovarieties. In particular, the trivial pseudovariety I and the pseudovariety $\mathrm{SI}$ of all finite semilattices are $\mathrm{H}$-solvable.

Notice that, if $M$ is a finite monoid and $\varphi: M \longrightarrow N$ is an onto homomorphism, we have $\varphi(E(M))=E(N)$ and so, in particular, $\langle\varphi(E(M))\rangle=\langle E(N)\rangle$. On the other hand, given monoids $M$ and $N$, it is clear that $E(M \times N)=E(M) \times E(N)$. It immediately follows that $\langle E(M \times N)\rangle \subseteq\langle E(M)\rangle \times\langle E(N)\rangle$. The converse inclusion is also true and easy to verify: we can write $m \in\langle E(M)\rangle$ and $n \in\langle E(N)\rangle$ as a product of idempotents 
with the same number of factors (just multiplying by 1 the shortest product, if one, as many times as necessary) and so we can write the pair $(m, n)$ as a product of elements of $E(M) \times E(N)$. Thus $\langle E(M \times N)\rangle=\langle E(M)\rangle \times\langle E(N)\rangle$. These observations are useful to prove the following result.

Proposition 2.2 For any pseudovariety $\mathrm{V}$ of monoids, the class $\mathrm{V}_{\mathrm{Hsol}}$ is closed under homomorphic images and finitary direct products.

Proof. Let $M, N \in \mathrm{V}_{\text {Hsol. }}$ Let $m, n \in \mathbb{N}$ be such that $\mathrm{K}_{\mathrm{H}}^{(m)}(M)=\langle E(M)\rangle$ and $\mathrm{K}_{\mathrm{H}}^{(n)}(N)=\langle E(N)\rangle$. By Theorem 2.1.4 and the observation above, we have

$$
\mathrm{K}_{\mathrm{H}}^{(m+n)}(M \times N)=\mathrm{K}_{\mathrm{H}}^{(m+n)}(M) \times \mathrm{K}_{\mathrm{H}}^{(m+n)}(N)=\langle E(M)\rangle \times\langle E(N)\rangle=\langle E(M \times N)\rangle,
$$

whence $M \times N \in \mathrm{V}_{\text {Hsol }}$. Thus $\mathrm{V}_{\text {Hsol }}$ is closed under finitary direct products.

Analogously, given a monoid $M \in \mathrm{V}_{\mathrm{Hsol}}$ and an onto homomorphism $\varphi: M \longrightarrow N$, since $N \in \mathrm{V}$ and in view of Theorem 2.1.3, it is easy to show that $N \in \mathrm{V}_{\text {Hsol }}$. Thus $\mathrm{V}_{\text {Hsol }}$ is also closed under homomorphic images.

Denote by $M$ and $G$ the pseudovarieties of all finite monoids and all finite groups respectively. Observe that, by Corollary $1.4, \mathrm{G}_{\text {sol }}$ is the class of all finite solvable groups. It is a pseudovariety of monoids. The class $\bar{G}_{\text {sol }}$ of all finite monoids whose subgroups are solvable is a pseudovariety largely considered in the literature (see, for instance, $[25,8]$ ) and its members are sometimes called solvable monoids. The search for relations between the two classes $\bar{G}_{\text {sol }}$ and $M_{\text {sol }}$ containing $G_{\text {sol }}$ or even relations between the intersection of these classes with some pseudovariety of monoids seems natural.

The class $M_{\text {sol }}$ is not contained in $\bar{G}_{\text {sol }}$ as is shown by the following computations carried out with GAP [27]. Let $T_{6}$ be the monoid of all total transformations on a set with 6 elements and let $S T_{6}$ be the submonoid consisting of the identity and the transformations of rank not greater than 5 (i.e. the transformations not belonging to the symmetric group $S_{6}$ ). Of course, $S T_{6}$ contains a copy of the symmetric group $S_{5}$, which is well-known to be non-solvable. On the other hand, the computations show that $S T_{6}$ is generated by idempotents thus $S T_{6}$ is its own Abelian kernel (in fact, this property is valid for transformations on any set with $n \geq 2$ elements [13]). Therefore $S T_{6} \in \mathrm{M}_{\text {sol }}$, but $S T_{6} \notin \overline{\mathrm{G}}_{\text {sol }}$ since it contains the non-solvable group $S_{5}$.

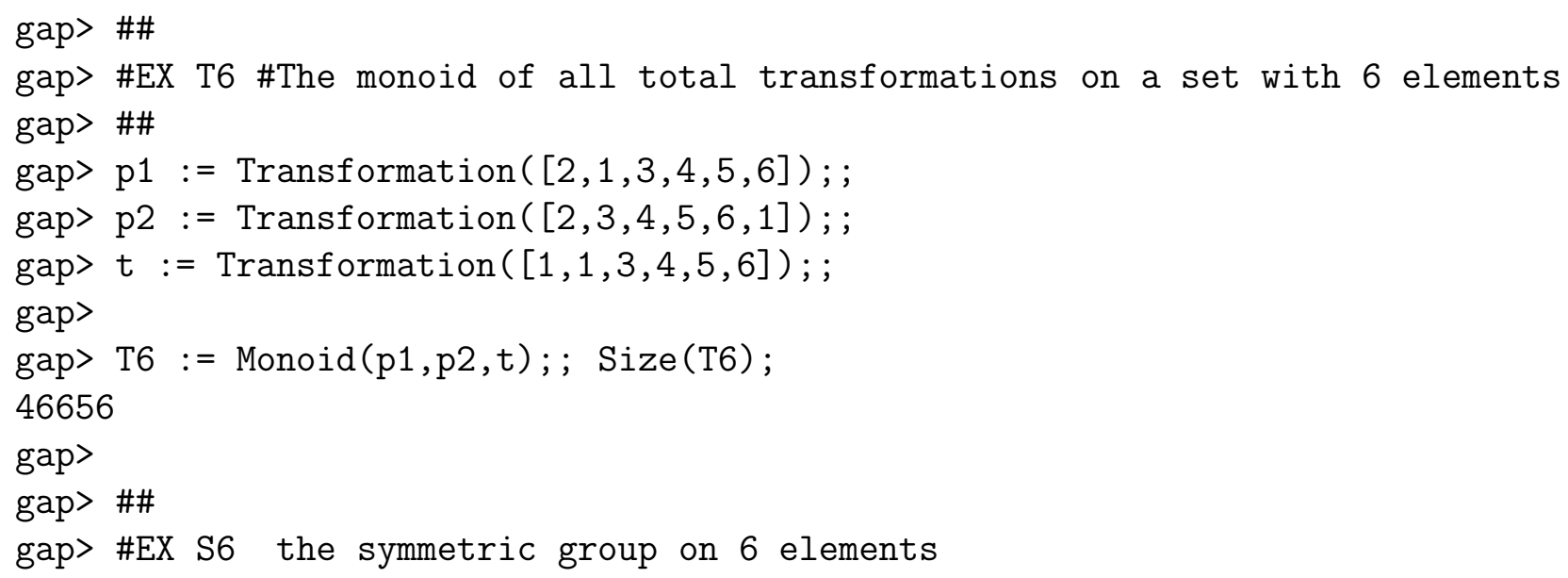




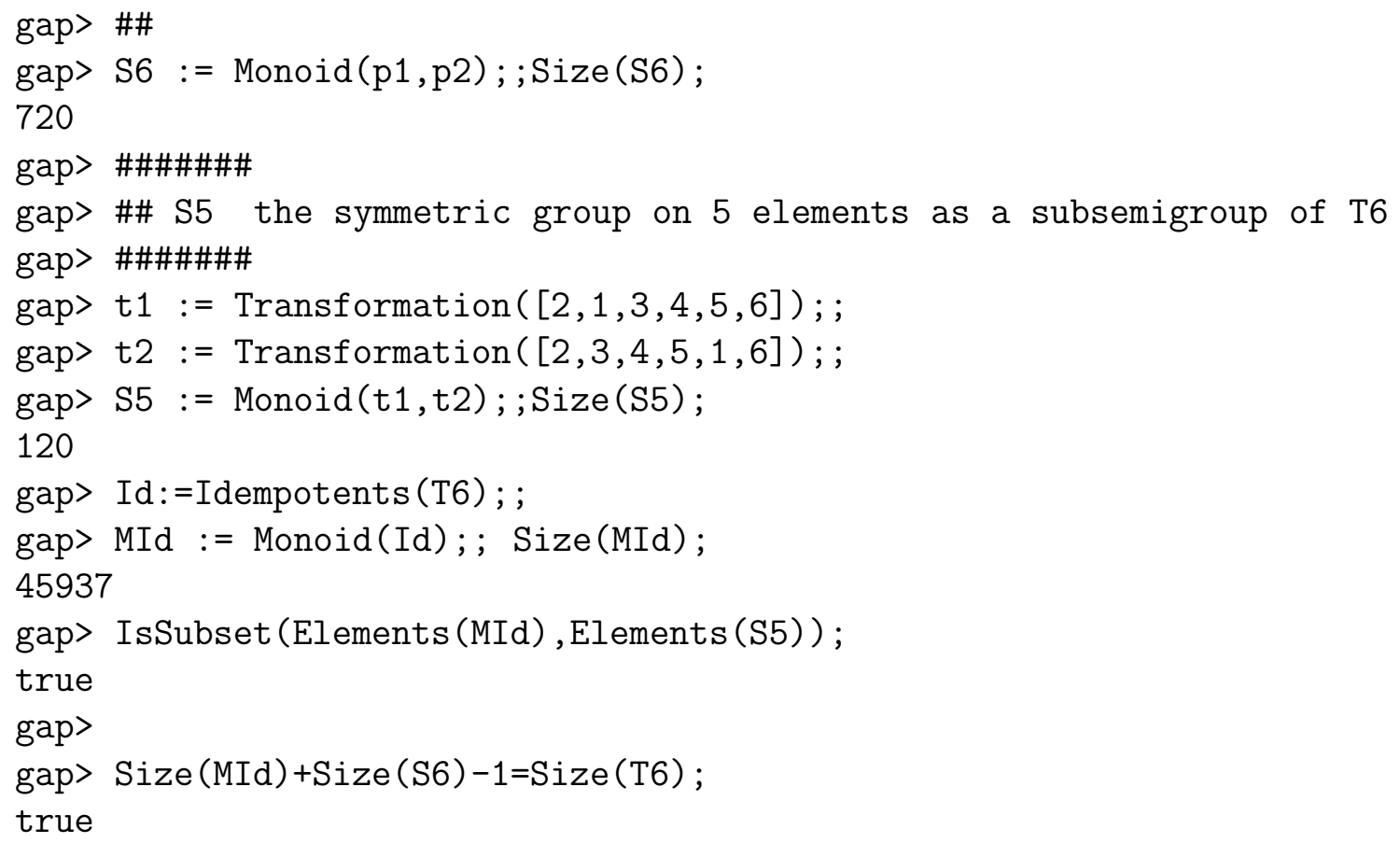

Notice that these computations also show that the class $M_{\text {sol }}$ is not a pseudovariety, since it is not closed for submonoids. This is no longer the situation when we consider monoids whose idempotents form a band. In this case, considering, more generally, any pseudovariety $\mathrm{H}$ of groups and denoting by $\overline{\mathrm{G}}_{\mathrm{Hsol}}$ the class of all finite monoids whose subgroups are in $\mathrm{G}_{\mathrm{Hsol}}$ and by $\mathrm{EB}$ the pseudovariety of monoids whose idempotents form a band, we have:

Proposition 2.3 Let $\mathrm{V} \subseteq \mathrm{EB}$ be a pseudovariety of monoids. Then $\mathrm{V}_{\mathrm{Hsol}}$ is a pseudovariety of monoids and $\mathrm{V}_{\mathrm{Hsol}} \subseteq \overline{\mathrm{G}}_{\mathrm{Hsol}} \cap \mathrm{V}$.

Proof. It suffices to prove that $\mathrm{V}_{\mathrm{Hsol}}$ is a pseudovariety of monoids. In view of Proposition 2.2, it remains to prove that $\mathrm{V}_{\mathrm{Hsol}}$ is closed under taking submonoids. Let $M \in \mathrm{V}_{\mathrm{Hsol}}$ and let $N$ be a submonoid of $M$. Suppose that $n \in \mathbb{N}$ is such that $\mathrm{K}_{\mathrm{H}}^{(n)}(M)=$ $E(M)$. Then $E(N) \subseteq \mathrm{K}_{\mathrm{H}}^{(n)}(N) \subseteq \mathrm{K}_{\mathrm{H}}^{(n)}(M) \cap N=E(M) \cap N=E(N)$, whence $N \in \mathrm{V}_{\text {Hsol }}$, as required.

Since $G \subseteq E B$, in particular, we have:

Corollary 2.4 The class $\mathrm{G}_{\mathrm{Hsol}}$ of all finite $\mathrm{H}$-solvable groups is a pseudovariety containing $\mathrm{H}$.

As a consequence of Corollary 2.4, we obtain that the class $\overline{\mathrm{G}}_{\mathrm{Hsol}}$ is also a pseudovariety of monoids (see [1, page 131]).

An important subpseudovariety of EB is Ecom, the class of all finite idempotent commuting monoids. In Section 3, we will prove that, for $\mathrm{V}=$ Ecom and $\mathrm{H}=\mathrm{Ab}$, the reverse of the inclusion stated in Proposition 2.3 also holds, i.e. we will prove that Ab-solvable 
idempotent commuting monoids are precisely the idempotent commuting monoids whose subgroups are solvable: Ecom $_{\text {sol }}=\overline{\mathrm{G}}_{\text {sol }} \cap$ Ecom.

Proposition 2.3 can be stated, not only for pseudovarieties of monoids whose idempotents form a band, but in fact, it can be proved more generally for any class of finite monoids whose idempotents generate a monoid belonging to a certain $\mathrm{H}$-solvable pseudovariety. Indeed, denoting by EV the pseudovariety of monoids whose idempotents generate a monoid in the pseudovariety $\mathrm{V}$, we have:

Theorem 2.5 Let $\mathrm{W}$ be a pseudovariety of $\mathrm{H}$-solvable monoids and let $\mathrm{V}$ be a subpseudovariety of $\mathrm{EW}$. Then $\mathrm{V}_{\mathrm{Hsol}}$ is a pseudovariety of monoids and $\mathrm{V}_{\mathrm{Hsol}} \subseteq \overline{\mathrm{G}}_{\mathrm{Hsol}} \cap \mathrm{V}$.

Proof. As in the proof of Proposition 2.3, it suffices to prove that $\mathrm{V}_{\mathrm{Hsol}}$ is closed under taking submonoids. Let $M \in \mathrm{V}_{\mathrm{Hsol}}$ and let $N$ be a submonoid of $M$. Let $n \in \mathbb{N}$ be such that $\mathrm{K}_{\mathrm{H}}^{(n)}(M)=\langle E(M)\rangle$. Then $\mathrm{K}_{\mathrm{H}}^{(n)}(N) \subseteq \mathrm{K}_{\mathrm{H}}^{(n)}(M)=\langle E(M)\rangle \in \mathrm{W}$, whence $\mathrm{K}_{\mathrm{H}}^{(n)}(N)$ is also a $\mathrm{H}$-solvable monoid and so there exists $m \in \mathbb{N}$ such that $\mathrm{K}_{\mathrm{H}}^{(m)}\left(\mathrm{K}_{\mathrm{H}}^{(n)}(N)\right)=$ $\left\langle E\left(\mathrm{~K}_{\mathrm{H}}^{(n)}(N)\right)\right\rangle$. Since $\left\langle E\left(\mathrm{~K}_{\mathrm{H}}^{(n)}(N)\right)\right\rangle=\langle E(N)\rangle$, it follows that $\mathrm{K}_{\mathrm{H}}^{(m+n)}(N)=\langle E(N)\rangle$, i.e. $N \in \mathrm{V}_{\mathrm{Hsol}}$, as required.

We have already observed that both Ecom $_{\mathrm{Hsol}}$ and $\mathrm{G}_{\mathrm{Hsol}}$ are pseudovarieties, but note that the same facts also follow directly from Theorem 2.5, since Ecom $=\mathrm{ESI}$ and $\mathrm{G}=\mathrm{EI}$.

\subsection{Regularity}

As usual, we denote by $\operatorname{Reg}(M)$ the set of all regular elements of the monoid $M$. The main result of this section shows that $M$ is $\mathrm{H}$-solvable if and only if $\langle\operatorname{Reg}(M)\rangle$ is $\mathrm{H}$-solvable.

Lemma 2.6 Let $M$ be a finite monoid. Let $x, y \in M$ be such that $x \mathcal{R} y$ (resp. $x \mathcal{L} y$ ). Then, there exist $u, v \in \operatorname{Reg}(M)$ such that $x=y u$ and $y=x v$ (resp. $x=$ uy and $y=v x$ ) and $u \mathrm{~J} v$.

Proof. Since $x \mathcal{R} y$, we can take $u \in M$ such that $x=y u$. Additionally, we may suppose that $J_{u}$ is a $\leq_{\mathcal{g}}$-minimal element of $\left\{J_{u^{\prime}} \mid u^{\prime} \in M\right.$ and $\left.x=y u^{\prime}\right\}$. Similarly, let us take

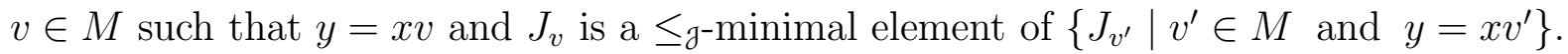
Since $x=y u=x v u=y \cdot u v u, y=x v=y u v=x \cdot v u v, J_{u v u} \leq_{\mathfrak{J}} J_{u}$ and $J_{v u v} \leq_{\mathfrak{J}} J_{v}$, we have $J_{u v u}=J_{u}$ and $J_{v u v}=J_{v}$, by the minimality of $J_{u}$ and $J_{v}$. Then $J_{u}=J_{u v u} \leq_{\mathcal{J}}$ $J_{v}=J_{v u v} \leq_{\mathfrak{\jmath}} J_{u v} \leq_{\mathfrak{\jmath}} J_{u}$, whence $J_{u}=J_{v}=J_{u v}$ and so, as $M$ is a finite, $u$ and $v$ must be regular elements.

It follows immediately that:

Lemma 2.7 Let $M$ be a finite monoid. Let $x, y \in M$ be such that $x \notin\langle\operatorname{Reg}(M)\rangle$. If $x \mathcal{R} y$ or $x \mathcal{L} y$ then $y \notin\langle\operatorname{Reg}(M)\rangle$.

And, consequently:

Proposition 2.8 Let $M$ be a finite monoid and let $x$ be an element of $M$. If $x \notin$ $\langle\operatorname{Reg}(M)\rangle$ then $J_{x} \cap\langle\operatorname{Reg}(M)\rangle=\emptyset$. 
Proposition 2.9 Let $M$ be a finite monoid such that $M \backslash\langle\operatorname{Reg}(M)\rangle \neq \emptyset$. Let $J$ be $a \leq_{\mathrm{g}-}$ maximal $\mathcal{J}$-class among the $\mathcal{J}$-classes of the elements of $M \backslash\langle\operatorname{Reg}(M)\rangle$. Then $N=M \backslash J$ is a submonoid of $M$ and $\operatorname{Reg}(N)=\operatorname{Reg}(M)$.

Proof. Take $x, y \in N$. If $x y \in\langle\operatorname{Reg}(M)\rangle$ then $x y \notin J$ and so $x y \in N$. On the other hand, suppose that $x y \notin\langle\operatorname{Reg}(M)\rangle$. Hence, $x \notin\langle\operatorname{Reg}(M)\rangle$ or $y \notin\langle\operatorname{Reg}(M)\rangle$. If $x y \in J$ then $J \leq_{\mathfrak{J}} J_{x}$ and $J \leq_{\mathfrak{J}} J_{y}$ and so, by the maximality of $J$, if $x \notin\langle\operatorname{Reg}(M)\rangle$ then $x \in J$ and if $y \notin\langle\operatorname{Reg}(M)\rangle$ then $y \in J$. Thus, since $x, y \notin J$, we must have $x y \notin J$, whence $x y \in N$. We have therefore proved that $N$ is a submonoid of $M$ (notice that $1 \in \operatorname{Reg}(M)$, whence $1 \in N)$.

Clearly, $\operatorname{Reg}(N) \subseteq \operatorname{Reg}(M)$. On the other hand, as $J$ is a non-regular $\mathcal{J}$-class of $M$, $\operatorname{Reg}(M) \subseteq N$ and so we have the reverse inclusion. $\operatorname{Thus} \operatorname{Reg}(N)=\operatorname{Reg}(M)$, as required.

Let $M$ be a finite monoid and let $J$ be a $\mathcal{J}$-class of $M$ as in previous proposition. Let $G$ be a non-trivial group and fix $g \in G \backslash\{1\}$. Define a relation $\tau: M \longrightarrow G$ by:

$$
\tau(x)= \begin{cases}\{1\} & \text { if } J<_{\mathfrak{g}} J_{x} \\ \{g\} & \text { if } x \in J \\ G & \text { otherwise (i.e. if } J \not \mathbb{g}_{\mathfrak{g}} J_{x} \text { ), }\end{cases}
$$

for all $x \in M$.

Next, we prove that $\tau$ is a relational morphism.

Let $x, y \in M$. If $J \not \mathbb{Z}_{\mathfrak{J}} J_{x y}$, we have $\tau(x) \tau(y) \subseteq G=\tau(x y)$. So, suppose that $J \leq_{\mathfrak{g}} J_{x y}$. Then, as $J$ is non-regular, $x \notin J$ or $y \notin J$. If $J<_{\mathfrak{g}} J_{x}$ and $J<_{\mathfrak{g}} J_{y}$, by the maximality of $J$, we must have $x, y \in\langle\operatorname{Reg}(M)\rangle$, whence $x y \in\langle\operatorname{Reg}(M)\rangle$ and so $x y \notin J$. In this case, $1 \in \tau(x y)$ and then

$$
\tau(x) \tau(y)=\{1\}\{1\}=\{1\} \subseteq \tau(x y) .
$$

If $J<_{\mathfrak{g}} J_{x}$ and $y \in J$, since $J_{x y} \leq_{\mathfrak{\jmath}} J_{y}=J$, we have $g \in \tau(x y)$, whence

$$
\tau(x) \tau(y)=\{1\}\{g\}=\{g\} \subseteq \tau(x y) .
$$

Finally, the case $x \in J$ and $J<_{\mathfrak{g}} J_{y}$ is similar to the preceding one.

Thus $\tau: M \multimap G$ is a relational morphism. Since $J \cap \tau^{-1}(1)=\emptyset$ (in fact, $\tau^{-1}(1)=$ $M \backslash J)$, we have:

Lemma 2.10 Let $M$ be a finite monoid such that $M \backslash\langle\operatorname{Reg}(M)\rangle \neq \emptyset$. Let $J$ be $a \leq_{\mathfrak{g}^{-}}$ maximal J-class among the J-classes of the elements of $M \backslash\langle\operatorname{Reg}(M)\rangle$. If $\mathrm{H}$ is non-trivial then $J \cap \mathrm{K}_{\mathrm{H}}(M)=\emptyset$.

Proposition 2.11 Let $M$ be a finite monoid. If $\mathrm{H}$ is non-trivial then $\mathrm{K}_{\mathrm{H}}^{(n)}(M) \subseteq\langle\operatorname{Reg}(M)\rangle$, for $n \geq|M \backslash\langle\operatorname{Reg}(M)\rangle|$.

Proof. We prove by induction on $n$ the following property: given $n \in \mathbb{N}, \mathrm{K}_{\mathrm{H}}^{(n)}(M) \subseteq$ $\langle\operatorname{Reg}(M)\rangle$, for any monoid $M$ such that $n=|M \backslash\langle\operatorname{Reg}(M)\rangle|$. 
For $n=0$ the property is trivial. Consecutively, let $n \geq 1$ and suppose that the property holds for all $k \in\{0, \ldots, n-1\}$. Take a monoid $M$ such that $|M \backslash\langle\operatorname{Reg}(M)\rangle|=n$. Let $J$ be a $\leq_{\mathcal{J}}$-maximal $\mathcal{J}$-class among the $\mathcal{J}$-classes of the elements of $M \backslash\langle\operatorname{Reg}(M)\rangle$. Let $N=M \backslash J$. Then, by Proposition 2.9, $N$ is a (proper) submonoid of $M$ and $\operatorname{Reg}(N)=\operatorname{Reg}(M)$. Hence $\langle\operatorname{Reg}(N)\rangle=\langle\operatorname{Reg}(M)\rangle$ and so $k=|N \backslash\langle\operatorname{Reg}(N)\rangle|<n$. By the induction hypothesis, we have $\mathrm{K}_{\mathrm{H}}^{(k)}(N) \subseteq\langle\operatorname{Reg}(N)\rangle$, whence $\mathrm{K}_{\mathrm{H}}^{(n-1)}(N) \subseteq\langle\operatorname{Reg}(N)\rangle$. On the other hand, by Lemma 2.10, we have $\mathrm{K}_{\mathrm{H}}(M) \subseteq N$. Thus

$$
\mathrm{K}_{\mathrm{H}}^{(n)}(M)=\mathrm{K}_{\mathrm{H}}^{(n-1)}\left(\mathrm{K}_{\mathrm{H}}(M)\right) \subseteq \mathrm{K}_{\mathrm{H}}^{(n-1)}(N) \subseteq\langle\operatorname{Reg}(N)\rangle=\langle\operatorname{Reg}(M)\rangle,
$$

as required.

Now, we can prove the following result.

Theorem 2.12 Let $M$ be a finite monoid. If $\mathrm{H}$ is non-trivial then $M$ is $\mathrm{H}$-solvable if and only if $\langle\operatorname{Reg}(M)\rangle$ is $\mathrm{H}$-solvable.

Proof. First, suppose that $M$ is $\mathrm{H}$-solvable. Take $n \in \mathbb{N}$ such that $\mathrm{K}_{\mathrm{H}}^{(n)}(M)=\langle E(M)\rangle$. Since $E(M)=E(\langle\operatorname{Reg}(M)\rangle)$, we have

$$
\langle E(M)\rangle=\langle E(\langle\operatorname{Reg}(M)\rangle)\rangle \subseteq \mathrm{K}_{\mathrm{H}}^{(n)}(\langle\operatorname{Reg}(M)\rangle) \subseteq \mathrm{K}_{\mathrm{H}}^{(n)}(M)=\langle E(M)\rangle,
$$

whence $\mathrm{K}_{\mathrm{H}}^{(n)}(\langle\operatorname{Reg}(M)\rangle)=\langle E(\langle\operatorname{Reg}(M)\rangle)\rangle$ and so $\langle\operatorname{Reg}(M)\rangle$ is $\mathrm{H}$-solvable.

On the other hand, suppose that $\langle\operatorname{Reg}(M)\rangle$ is $\mathrm{H}$-solvable and let $n \in \mathbb{N}$ be such that

$$
\mathrm{K}_{\mathrm{H}}^{(n)}(\langle\operatorname{Reg}(M)\rangle)=\langle E(\langle\operatorname{Reg}(M)\rangle)\rangle .
$$

Applying Proposition 2.11, we can take a non-negative integer $m$ such that $\mathrm{K}_{\mathrm{H}}^{(m)}(M) \subseteq$ $\langle\operatorname{Reg}(M)\rangle$. Then

$\langle E(M)\rangle \subseteq \mathrm{K}_{\mathrm{H}}^{(n+m)}(M)=\mathrm{K}_{\mathrm{H}}^{(n)}\left(\mathrm{K}_{\mathrm{H}}^{(m)}(M)\right) \subseteq \mathrm{K}_{\mathrm{H}}^{(n)}(\langle\operatorname{Reg}(M)\rangle)=\langle E(\langle\operatorname{Reg}(M)\rangle)\rangle=\langle E(M)\rangle$,

whence $\mathrm{K}_{\mathrm{H}}^{(n+m)}(M)=\langle E(M)\rangle$ and so $M$ is $\mathrm{H}$-solvable, as required.

Corollary 2.13 Let $M \in \mathrm{Ecom}$. If $\mathrm{H}$ is non-trivial then $M$ is $\mathrm{H}$-solvable if and only if (its inverse submonoid) $\operatorname{Reg}(M)$ is $\mathrm{H}$-solvable.

\subsection{Monoids in DA}

Let DA be the pseudovariety consisting of all finite monoids whose regular elements are idempotents and let $M \in \mathrm{DA}$. Applying Proposition 2.11, we have

$$
E(M) \subseteq \mathrm{K}_{\mathrm{H}}^{(n)}(M) \subseteq\langle\operatorname{Reg}(M)\rangle=\langle E(M)\rangle,
$$

for some $n \in \mathbb{N}$, whence $\mathrm{K}_{\mathrm{H}}^{(n)}(M)=\langle E(M)\rangle$, i.e. $M$ is $\mathrm{H}$-solvable. Thus, we have proved the following result.

Theorem 2.14 If $\mathrm{H}$ is non-trivial and $\mathrm{V}$ is a pseudovariety of monoids contained in DA, then $\mathrm{V}$ is $\mathrm{H}$-solvable. 
By applying Theorem 2.5, we obtain the following corollary, which, since $\mathrm{B} \subseteq \mathrm{DA}$, generalises Proposition 2.3:

Corollary 2.15 If $\mathrm{H}$ is non-trivial and $\mathrm{V}$ is a pseudovariety of monoids contained in $\mathrm{DA}$, then the class $\mathrm{EV}_{\mathrm{Hsol}}$ is a pseudovariety of monoids such that $\mathrm{EV}_{\mathrm{Hsol}} \subseteq \overline{\mathrm{G}}_{\mathrm{Hsol}} \cap \mathrm{EV}$.

We remark that, in addition to $B$, there are other interesting subpseudovarieties of DA to which our previous results apply. Among them, we can refer J, the pseudovariety of all $\partial$-trivial monoids.

\section{Solvable monoids with commuting idempotents}

In this section we prove that, inside the class of all finite monoids with commuting idempotents, the notion of $\mathrm{Ab}$-solvable monoid coincides with the notion of monoid whose subgroups are solvable, i.e. in terms of pseudovarieties: Ecom $_{\text {sol }}=\bar{G}_{\text {sol }} \cap$ Ecom. We first prove this equality for inverse monoids and then extend it to all idempotent commuting monoids.

As already observed, the $\mathrm{H}$-kernel of a finite monoid is a submonoid containing the idempotents. In the case of an inverse monoid we can say more:

Proposition 3.1 The $\mathrm{H}$-kernel of a finite inverse monoid $M$ is an inverse submonoid of $M$.

Proof. Let $x \in \mathrm{K}_{\mathrm{H}}(M)$. It suffices to observe that, for any relational morphism $\tau: M \multimap G$ into a group $G \in \mathrm{H}, 1 \in \tau\left(x^{-1}\right)$. Since $1 \in \tau(x)$, we have $\tau\left(x^{-1}\right) \subseteq$ $\tau(x) \tau\left(x^{-1}\right) \subseteq \tau\left(x x^{-1}\right)$. Thus $\tau\left(x^{-1}\right) \cdot \tau\left(x^{-1}\right) \subseteq \tau\left(x^{-1}\right) \tau\left(x x^{-1}\right) \subseteq \tau\left(x^{-1} x x^{-1}\right)=\tau\left(x^{-1}\right)$. So $\tau\left(x^{-1}\right)$ is a non-empty subset of the finite group $G$ which is closed under multiplication and therefore is a subgroup of $G$. Thus $\tau\left(x^{-1}\right)$ contains 1 , as required.

Recall that given a semigroup $S$, a $\mathcal{D}$-class $D$ of $S$ and elements $a, b \in D$, we have that $R_{a} \cap L_{b}$ is an $\mathcal{H}$-class of $S$. Moreover, $a b \in R_{a} \cap L_{b}$ if and only if $L_{a} \cap R_{b}$ contains an idempotent (see [18] for details). Furthermore, if $S$ is finite then $a b \in D$ if and only if $a b \in R_{a} \cap L_{b}$. In fact, since in a finite semigroup the restrictions of the relations $\leq_{\mathcal{R}}$ and $\leq_{\mathcal{L}}$ to a $\mathcal{D}$-class are trivial (see [20]) and $a b \leq_{\mathcal{R}} a$ and $a b \leq_{\mathcal{L}} b$, if $a b \in D$ then $a b \mathcal{R} a$ and $a b \mathcal{L} b$, i.e. $a b \in R_{a} \cap L_{b}$. The converse is trivial.

Let $M$ be a finite inverse monoid and let $J$ be a J-class of $M$. Denote by $\langle J\rangle$ the submonoid of $M$ generated by $J$. Notice that $\langle J\rangle$ is (also) an inverse monoid.

Note 1 Consider an element $x \in J$ and an idempotent $e \in M$. If $e x \in J$ then, since $M$ is finite, we have $e x \mathcal{L} x$, whence $(e x)^{-1} e x=x^{-1} x$ and so

$$
x=x x^{-1} x=x(e x)^{-1} e x=x x^{-1} e e x=x x^{-1} e x=e x x^{-1} x=e x .
$$

Similarly, if $x e \in J$ then $x e=x$. 
Lemma 3.2 Let $M$ be a finite inverse monoid. If $J$ is a non-trivial J-class of $M$ which

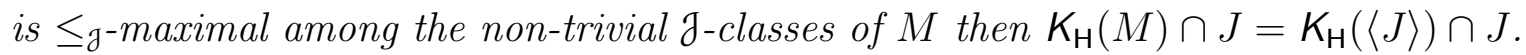

Proof. First, notice that, since $\langle J\rangle$ is a submonoid of $M, \mathrm{~K}_{\mathrm{H}}(M) \supseteq \mathrm{K}_{\mathrm{H}}(\langle J\rangle)$ and so $\mathrm{K}_{\mathrm{H}}(M) \cap J \supseteq \mathrm{K}_{\mathrm{H}}(\langle J\rangle) \cap J$.

Conversely, let $x \in \mathrm{K}_{\mathrm{H}}(M) \cap J$ and let $\tau:\langle J\rangle \rightarrow G$ be a relational morphism, with $G \in \mathrm{H}$. Let us consider the extension $\tilde{\tau}$ of $\left.\tau\right|_{J}$ to $M$ defined by:

$$
\tilde{\tau}(m)=\left\{\begin{array}{cl}
\{1\} & \text { if } J<_{\mathfrak{g}} J_{m} \\
\tau(m) & \text { if } m \in J \\
G & \text { if } J \mathbb{Z}_{\mathfrak{J}} J_{m} .
\end{array}\right.
$$

Next, we prove that $\tilde{\tau}: M \rightarrow G$ is also a relational morphism. Let $a, b \in M$. If $J \not \mathbb{z}_{\mathrm{J}} J_{a b}$ then $\tilde{\tau}(a) \tilde{\tau}(b) \subseteq G=\tilde{\tau}(a b)$. So, suppose that $J \leq_{\mathcal{J}} J_{a b}$. If $a b \notin J$ then $a, b \notin J$, whence $\tilde{\tau}(a) \tilde{\tau}(b)=\{1\}\{1\}=\{1\}=\tilde{\tau}(a b)$. Let us now suppose that $a b \in J$. If $a, b \in J$ then $\tilde{\tau}(a) \tilde{\tau}(b)=\tau(a) \tau(b) \subseteq \tau(a b)=\tilde{\tau}(a b)$. If $J<_{\mathfrak{J}} J_{a}$ (whence $a^{2}=a$ and $J_{a}=\{a\}$ ) and $b \in J$, by Note 1 , we have $a b=b$ and so $\tilde{\tau}(a) \tilde{\tau}(b)=\{1\} \tau(b)=\tau(b)=\tilde{\tau}(b)=\tilde{\tau}(a b)$. Finally, the case $a \in J$ and $J<_{\mathfrak{g}} J_{b}$ is similar to the precedent one.

As $x \in \mathrm{K}_{\mathrm{H}}(M) \cap J, 1 \in \tilde{\tau}(x)=\tau(x)$, i.e. $x \in \tau^{-1}(1)$. Thus $x \in \mathrm{K}_{\mathrm{H}}(\langle J\rangle)$ and so $\mathrm{K}_{\mathrm{H}}(M) \cap J \subseteq \mathrm{K}_{\mathrm{H}}(\langle J\rangle) \cap J$, as required.

Let $e_{1}, \ldots, e_{n}$ be all the distinct idempotents of $J$.

Consider the maximal subgroup $H_{e_{1}}$ of $M$, contained in the $\mathcal{J}$-class $J$, and fix elements $a_{2}, \ldots, a_{n}$ in the $\mathcal{R}$-class of $e_{1}$ such that $a_{i} \in L_{e_{i}}$, for $2 \leq i \leq n$. Then, observe that $a_{i}^{-1} \in R_{e_{i}} \cap L_{e_{1}}$, for $2 \leq i \leq n$. The following egg-box picture may help to visualise the situation.

\begin{tabular}{|c|c|c|c|}
\hline$e_{1}$ & $a_{2}$ & $\cdots$ & $a_{n}$ \\
\hline$a_{2}^{-1}$ & $e_{2}$ & $\cdots$ & \\
\hline$\vdots$ & $\vdots$ & & $\vdots$ \\
\hline$a_{n}^{-1}$ & & $\cdots$ & $e_{n}$ \\
\hline
\end{tabular}

Let $a_{1}=e_{1}$. By Green's Lemma, the mappings

$$
\begin{array}{ccc}
R_{a_{i}^{-1}} \cap L_{a_{j}} & \longrightarrow & H_{e_{1}} \\
x & \mapsto & a_{i} x a_{j}^{-1},
\end{array}
$$

with $1 \leq i, j \leq n$, are bijections (in fact, isomorphisms when $i=j$ ) and induce, naturally, a function $\Phi: J \rightarrow H_{e_{1}}$ such that the restriction to $H_{e_{1}}$ is the identity.

Let $x, y \in J$. Then $x \in R_{a_{i}^{-1}} \cap L_{a_{r}}$ and $y \in R_{a_{s}^{-1}} \cap L_{a_{j}}$, for some $1 \leq i, j, r, s \leq n$. Suppose that $x y \in J$. Then $x y \in R_{x} \cap L_{y}$ and $L_{x} \cap R_{y}$ is a subgroup of $M$, i.e. $x y \in R_{a_{i}^{-1}} \cap$ $L_{a_{j}}$ and $r=s$. Since $x^{-1} x=a_{r}^{-1} a_{r}$, we have $x=x a_{r}^{-1} a_{r}$ and so $a_{i} x y a_{j}^{-1}=a_{i} x a_{r}^{-1} a_{r} y a_{j}^{-1}$, i.e.

$$
\Phi(x y)=\Phi(x) \Phi(y) .
$$

Now, we can prove the following lemma:

Lemma 3.3 Let $M$ be a finite inverse monoid and let $J$ be a non-trivial J-class of $M$

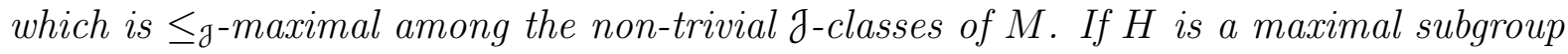
of $M$ contained in $J$ then $\mathrm{K}_{\mathrm{H}}(M) \cap H=\mathrm{K}_{\mathrm{H}}(H)$ and, in particular, $\mathrm{K}_{\mathrm{Ab}}(M) \cap H=H^{\prime}$. 
Proof. With the notation introduced above, we may suppose that $H=H_{e_{1}}$.

Since $H$ is a subgroup of $M$, we immediately have $\mathrm{K}_{\mathrm{H}}(H) \subseteq \mathrm{K}_{\mathrm{H}}(M) \cap H$.

To prove the converse inclusion, let $x \in \mathrm{K}_{\mathrm{H}}(M) \cap H$ and let $\tau: H \multimap G$ be a relational morphism from $H$ into a group $G \in \mathrm{H}$. Define an extension $\tilde{\tau}: M \multimap G$ of $\tau$ as follows:

$$
\tilde{\tau}(x)= \begin{cases}\{1\} & \text { if } J<_{\mathfrak{J}} J_{x} \\ \tau(\Phi(x)) & \text { if } x \in J \\ G & \text { if } J \mathbb{Z}_{\mathfrak{J}} J_{x} .\end{cases}
$$

We prove that $\tilde{\tau}$ is a relational morphism.

Let $x, y \in M$.

First, notice that if $J \not_{\mathcal{J}} J_{x y}$ then it is clear that $\tilde{\tau}(x) \tilde{\tau}(y) \subseteq G=\tilde{\tau}(x y)$. Hence, we may suppose that $J \leq_{\mathcal{J}} J_{x y}$ and so $J \leq_{\mathcal{J}} J_{x}$ and $J \leq_{\mathcal{J}} J_{y}$.

If $J<_{\mathfrak{f}} J_{x}$ and $J<_{\mathfrak{f}} J_{y}$, then $J_{x}$ and $J_{y}$ are trivial by the definition of $J$. Therefore, $x, y$ are idempotent, whence $x y$ is idempotent since $M$ is an inverse monoid. If $J<\mathfrak{\jmath} J_{x y}$ then $\tilde{\tau}(x y)=\{1\}=\tilde{\tau}(x) \tilde{\tau}(y)$. On the other hand, if $x y \in J$ then we have $\Phi(x y)=e_{1}$ and so $\tilde{\tau}(x) \tilde{\tau}(y)=\{1\} \subseteq \tau\left(e_{1}\right)=\tau(\Phi(x y))=\tilde{\tau}(x y)$.

If $x \in J$ and $J<_{\mathfrak{g}} J_{y}$ then $x y \in J$ and so, by Note $1, x y=x$, whence

$$
\tilde{\tau}(x) \tilde{\tau}(y)=\tilde{\tau}(x)\{1\}=\tilde{\tau}(x)=\tilde{\tau}(x y) .
$$

Similarly, if $J<_{\mathcal{J}} J_{x}$ and $y \in J$ then $\tilde{\tau}(x) \tilde{\tau}(y)=\tilde{\tau}(x y)$.

Finally, if $x, y \in J$ then $x y \in J$ and

$$
\tilde{\tau}(x) \tilde{\tau}(y)=\tau(\Phi(x)) \tau(\Phi(y)) \subseteq \tau(\Phi(x) \Phi(y))=\tau(\Phi(x y))=\tilde{\tau}(x y) .
$$

Then $\tilde{\tau}$ is, in fact, a relational morphism and, as $x \in \mathrm{K}_{\mathrm{H}}(M) \cap H$, we have $1 \in \tilde{\tau}(x)=$ $\tau(\Phi(x))=\tau(x)$, i.e. $x \in \tau^{-1}(1)$. Thus $x \in \mathrm{K}_{\mathbf{H}}(H)$, as required.

Let $x_{1}, \ldots, x_{n}$ be elements of $J$ such that $x_{i} \in R_{e_{i}} \cap L_{e_{i+1}}$, for $1 \leq i \leq n-1$, and $x_{n} \in R_{e_{n}} \cap L_{e_{1}}$. Let $Y$ be a set of generators of $H_{e_{n}}$. The situation is sketched in the following egg-box picture.

\begin{tabular}{|c|c|c|c|c|}
\hline$e_{1}$ & $x_{1}$ & & $\cdots$ & \\
\hline & $e_{2}$ & $x_{2}$ & $\cdots$ & \\
\hline$\vdots$ & $\vdots$ & $\vdots$ & & $\vdots$ \\
\hline & & & $\cdots$ & $x_{n-1}$ \\
\hline$x_{n}$ & & & $\cdots$ & $e_{n}, Y$ \\
\hline
\end{tabular}

Notice that, given $i, j \in\{1, \ldots, n\}$, since $x_{i} x_{j} \in J$ if and only if $L_{x_{i}} \cap R_{x_{j}}$ contains an idempotent, we have $x_{i} x_{j} \in J$ if and only if

$$
j=\left\{\begin{array}{cl}
i+1 & \text { if } 1 \leq i \leq n-1 \\
1 & \text { if } i=n
\end{array}\right.
$$

(whence $x_{i} x_{i+1} \in R_{x_{i}} \cap L_{x_{i+1}}$, for $1 \leq i \leq n-1$, and $x_{n} x_{1} \in R_{x_{n}} \cap L_{x_{1}}$ ). Moreover, it is a routine matter to prove that

$$
x_{i} \cdots x_{n} x_{1} \cdots x_{j} \in R_{x_{i}} \cap L_{x_{j}},
$$

for all $1 \leq i, j \leq n$. We thus obtain the following egg-box picture. 


\begin{tabular}{|c|c|c|c|c|}
\hline$x_{1} \cdots x_{n}, e_{1}$ & $x_{1}$ & $x_{1} x_{2}$ & $\cdots$ & $x_{1} \cdots x_{n-1}$ \\
\hline$x_{2} \cdots x_{n}$ & $x_{2} \cdots x_{n} x_{1}, e_{2}$ & $x_{2}$ & $\cdots$ & $x_{2} \cdots x_{n-1}$ \\
\hline$\vdots$ & $\vdots$ & $\vdots$ & & $\vdots$ \\
\hline$x_{n-1} x_{n}$ & $x_{n-1} x_{n} x_{1}$ & $x_{n-1} x_{n} x_{1} x_{2}$ & $\cdots$ & $x_{n-1}$ \\
\hline$x_{n}$ & $x_{n} x_{1}$ & $x_{n} x_{1} x_{2}$ & $\cdots$ & $x_{n} x_{1} \cdots x_{n-1}, e_{n}, Y$ \\
\hline
\end{tabular}

Next, we prove that $X=\left\{x_{1}, x_{2}, \ldots, x_{n}\right\} \cup Y$ generates $\langle J\rangle$. It suffices to show that each element of $R_{x_{i}} \cap L_{x_{j}}$ is a product of elements of $X$, for all $1 \leq i, j \leq n$. Let $i, j \in\{1,2, \ldots, n\}$. By Green's Lemma, the mapping

$$
\begin{array}{ccc}
H_{e_{n}} & \longrightarrow & R_{x_{i}} \cap L_{x_{j}} \\
x & \mapsto & x_{i} \cdots x_{n-1} x x_{n} x_{1} \cdots x_{j}
\end{array}
$$

is a bijection. Therefore, since any element of $H_{e_{n}}$ is a product of elements of $Y$, we obtain each element of $R_{x_{i}} \cap L_{x_{j}}$ as a product of elements of $X$, as required.

Now, let $\varphi: X^{*} \longrightarrow\langle J\rangle$ be the (unique) onto homomorphism extending the inclusion $\operatorname{map} X \hookrightarrow\langle J\rangle$. Then, we have

$$
\varphi^{-1}\left(x_{1}\right) \subseteq x_{1}\left(x_{2} \cdots x_{n-1} Y^{*} x_{n} x_{1}\right)^{*}
$$

In fact, let $z_{1}, z_{2}, \ldots, z_{m} \in X$ be such that $w=z_{1} z_{2} \cdots z_{m} \in \varphi^{-1}\left(x_{1}\right)$. Then $z_{1} \mathcal{R} x_{1} \mathcal{L} z_{m}$ and so $z_{1}=x_{1}=z_{m}$. Suppose that $m \geq 2$. It follows that

$$
w=u_{1} v_{1} u_{2} v_{2} \cdots u_{\ell} v_{\ell} u_{\ell+1}
$$

for some $\ell \geq 0, u_{1}, u_{2}, \ldots, u_{\ell}, u_{\ell+1} \in\left\{x_{1}, x_{2}, \ldots, x_{n}\right\}^{+}$such that $x_{1}$ is a prefix of $u_{1}$ and a suffix of $u_{\ell+1}$ and $v_{1}, v_{2}, \ldots, v_{\ell} \in Y^{+}$. Next, observe that, given $y \in H_{e_{n}}$ and $a \in J$ such that $a y \in J$, we must have $a \mathcal{L} y$. In fact, since $a y \in J$, then $a y \in R_{a} \cap L_{y}$ and so $L_{a} \cap R_{y}$ is a subgroup, whence $L_{a} \cap R_{y}=H_{e_{n}}$ and so $L_{a}=L_{e_{n}}=L_{y}$, as required. Analogously, if $y \in H_{e_{n}}$ and $b \in J$ are such that $y b \in J$ then $b \mathcal{R} y$. Thus, given $y \in Y$ and $x \in X, x y \in J$ implies $x \in Y \cup\left\{x_{n-1}\right\}$ and $y x \in J$ implies $x \in Y \cup\left\{x_{n}\right\}$. Therefore, we can conclude that $x_{n-1}$ is a suffix of $u_{1}, u_{2}, \ldots, u_{\ell}$ and $x_{n}$ is a prefix of $u_{2}, \ldots, u_{\ell+1}$. Now, in view of (2), it follows that $u_{1} \in x_{1}\left(x_{2} \cdots x_{n} x_{1}\right)^{*} x_{2} \cdots x_{n-1}, u_{2}, \ldots, u_{\ell} \in\left(x_{n} x_{1} \cdots x_{n-1}\right)^{+}$and $u_{\ell+1} \in$ $x_{n} x_{1}\left(x_{2} \cdots x_{n} x_{1}\right)^{*}$, which allow us to deduce easily that $w \in x_{1}\left(x_{2} \cdots x_{n-1} Y^{*} x_{n} x_{1}\right)^{*}$. Hence, we have proved (3).

Proposition 3.4 Let $M$ be a finite inverse monoid. Let $J$ be a non-trivial J-class of $M$.

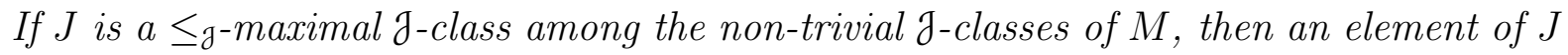
belongs to the Abelian kernel of $M$ if and only if it belongs to the derived subgroup of a maximal subgroup of $M$.

Proof. As the Abelian kernel of a monoid contains the Abelian kernel of a monoid that is a subsemigroup of the monoid, an element of $J$ that belongs to the derived subgroup of a maximal subgroup of $M$ also belongs to $\mathrm{K}_{\mathrm{Ab}}(M)$.

To prove the converse, we prove that an element of $J$ belonging to the Abelian kernel of $M$ also belongs to a (maximal) subgroup of $M$ and so, by Lemma 3.3, it follows that this element must belong to the derived subgroup of a maximal subgroup of $M$. 
Let $x_{1} \in J$ and suppose that $x_{1}$ does not belong to a subgroup of $M$. Then, by Lemma 3.2 , it suffices to prove that $x_{1}$ does not belong to $\mathrm{K}_{\mathrm{Ab}}(\langle J\rangle)$.

Let $e_{1}, e_{2}, \ldots, e_{n}$ be all the distinct idempotents of $J$ (notice that $n \geq 2$, as $x_{1}$ does not belong to a subgroup of $M$ ) and suppose that $e_{1}=x_{1} x_{1}^{-1}$ and $e_{2}=x_{1}^{-1} x_{1}$. Then $x_{1} \in R_{e_{1}} \cap L_{e_{2}}$. Also, let $x_{2}, \ldots, x_{n}$ be elements of $J$ such that $x_{i} \in R_{e_{i}} \cap L_{e_{i+1}}$, for $2 \leq i \leq n-1$, and $x_{n} \in R_{e_{n}} \cap L_{e_{1}}$. Let $Y=\left\{x_{n+1}, x_{n+2}, \ldots, x_{m}\right\}$ be a set of generators of $H_{e_{n}}$ and $X=\left\{x_{1}, x_{2}, \ldots, x_{n}\right\} \cup Y$. Then $X$ generates $\langle J\rangle$. Let $\varphi: X^{*} \longrightarrow\langle J\rangle$ be the onto homomorphism extending the the inclusion map $X \hookrightarrow\langle J\rangle$. Then, as observed above,

$$
\varphi^{-1}\left(x_{1}\right) \subseteq x_{1}\left(x_{2} \cdots x_{n-1} Y^{*} x_{n} x_{1}\right)^{*}
$$

and so

$$
\begin{aligned}
\overline{\gamma\left(\varphi^{-1}\left(x_{1}\right)\right)} \subseteq & (1,0, \ldots, 0)+\left(1, \ldots, 1_{(n)}, 0, \ldots, 0\right) \mathbb{Z}+\left(0, \ldots, 0,1_{(n+1)}, 0, \ldots, 0\right) \mathbb{Z}+ \\
& \left(0, \ldots, 0,1_{(n+2)}, 0, \ldots, 0\right) \mathbb{Z}+\cdots+(0, \ldots, 0,1) \mathbb{Z} .
\end{aligned}
$$

Thus, since $n \geq 2$, we deduce that $0 \notin \overline{\gamma\left(\varphi^{-1}\left(x_{1}\right)\right)}$, whence $x_{1}$ does not belong to $\mathrm{K}_{\mathrm{Ab}}(\langle J\rangle)$, as required.

Next, we can prove the following characterisation of the class of finite inverse Absolvable monoids.

Proposition 3.5 A finite inverse monoid is Ab-solvable if and only if all its subgroups are solvable.

Proof. As the class of all finite idempotent commuting Ab-solvable monoids is a pseudovariety, a finite inverse monoid only has solvable subgroups.

Conversely, let us suppose that there exists a non Ab-solvable inverse monoid with solvable subgroups and let $M$ be such a monoid of minimal cardinality. Since $M$ is not Ab-solvable, $\mathrm{K}_{\mathrm{Ab}}(M)$ is also not $\mathrm{Ab}$-solvable. On the other hand, as the subgroups of $M$ are solvable, then $\mathrm{K}_{\mathrm{Ab}}(M)$ has solvable subgroups. As, by Proposition $3.1, \mathrm{~K}_{\mathrm{Ab}}(M)$ is also an inverse monoid, by the minimality of $M$, we obtain $\mathrm{K}_{\mathrm{Ab}}(M)=M$. Since $M$ is not Ab-solvable, $M$ must have a non-trivial $\mathcal{J}$-class. Let $J$ be a non-trivial $\mathcal{J}$-class of $M$ which is $\leq \mathfrak{g}$-maximal among the non-trivial $\mathcal{J}$-classes of $M$. If $J$ is not a subgroup then, by Proposition 3.4, there exists an element of $J$ which does not belong to $\mathrm{K}_{\mathrm{Ab}}(M)$, a contradiction. Thus $J$ must be a subgroup and, applying Lemma 3.3, we have

$$
J=M \cap J=\mathrm{K}_{\mathrm{Ab}}(M) \cap J=\mathrm{K}_{\mathrm{Ab}}(J),
$$

which is also a contradiction, since $J$ is solvable and non-trivial.

Now, consider a monoid $M$ with commuting idempotents. Since the subgroups of $M$ are precisely the subgroups of its inverse submonoid $\operatorname{Reg}(M)$ (and so $M \in \overline{\mathrm{G}}_{\text {sol }}$ if and only if $\operatorname{Reg}(M) \in \overline{\mathrm{G}}_{\mathrm{sol}}$ ), by applying Corollary 2.13, with $\mathrm{H}=\mathrm{Ab}$, together with Proposition 3.5, we immediately obtain our main result:

Theorem 3.6 A finite monoid with commuting idempotents is Ab-solvable if and only if all its subgroups are solvable, i.e. $\mathrm{Ecom}_{\mathrm{sol}}=\overline{\mathrm{G}}_{\mathrm{sol}} \cap \mathrm{Ecom}$.

Corollary 3.7 Any finite aperiodic monoid with commuting idempotents is Ab-solvable. 


\section{Semidirect products by $\mathrm{H}$-solvable groups}

In this section we will consider Mal'cev and semidirect products of pseudovarieties of monoids by pseudovarieties of groups. We start recalling some well-known results concerning these operators and then proceed considering their iterations.

As already mentioned, for any pseudovariety $\mathrm{V}$ of monoids the inclusion $\mathrm{V} * \mathrm{H} \subseteq \mathrm{V} m \mathrm{H}$ holds. A sufficient condition for equality is: $\mathrm{V}$ is local in the sense of [28]. The condition is not necessary since, for instance, $\mathrm{J} * \mathrm{G}=\mathrm{J} \rightarrow \mathrm{G}$ and $\mathrm{J}$ is known to be non-local. This is one of the consequences of Ash's theorem referred to in [15]. Steinberg [24] has shown that the equality still holds for some other pseudovarieties of groups.

Pseudovarieties of finite groups (seen as pseudovarieties of monoids) are local, as is the pseudovariety A of all finite aperiodic monoids. (See, for example, [28].) However, the semidirect product of local pseudovarieties is not necessarily local. Moreover, this may even be the case when the second factor is a pseudovariety of groups. In order to justify this statement we need to recall the definition of complexity of a finite semigroup. According to the Krohn-Rhodes decomposition theorem, every finite semigroup $S$ belongs to some iterated semidirect product

$$
(\mathrm{A} * \mathrm{G})^{n} * \mathrm{~A}=\underbrace{(\mathrm{A} * \mathrm{G}) * \cdots *(\mathrm{~A} * \mathrm{G})}_{n \text { times }} * \mathrm{~A} .
$$

The group complexity of $S$ is the least non-negative integer $n$ such that $S \in(\mathrm{A} * \mathrm{G})^{n} * \mathrm{~A}$. Rhodes [21] exhibited an example of a complexity 2 semigroup in $(A * G)(m)$. Thus, $A * G$ cannot be local, otherwise $(A * G) \rightarrow G$ would be equal to $A * G * G$ which in turn is equal to $A * G$, but $A * G$ consists of semigroups whose group complexity is not greater than 1.

From the fact that any pseudovariety of groups is local follows that $\mathrm{I} *{ }^{n} \mathrm{H}=\mathrm{I}(m)^{n} \mathrm{H}$. Therefore, in view of Proposition 1.5, the class $\mathrm{G}_{\mathrm{Hsol}}$ of all finite $\mathrm{H}$-solvable groups is precisely

$$
\cup_{n \geq 1} \mathrm{I}(\mathrm{m})^{n} \mathrm{H}=\cup_{n \geq 1} \mathrm{I} *^{n} \mathrm{H}=\cup_{n \geq 1} \mathrm{H}^{n},
$$

where $\mathrm{H}^{n}$ denotes the semidirect power with $n$ factors of $\mathrm{H}$.

Let $\mathrm{V}$ be a pseudovariety of $\mathrm{H}$-solvable monoids. Then

$$
\mathrm{EV}_{\mathrm{Hsol}}=\cup_{n \geq 1} \mathrm{~V}()^{n} \mathrm{H}
$$

In fact, if $M \in \mathrm{EV}_{\mathrm{Hsol}}$ then $\mathrm{K}_{\mathrm{H}}^{(n)}(M)=\langle E(M)\rangle$, for some $n \geq 1$, and $\langle E(M)\rangle \in \mathrm{V}$. Hence $M \in \mathrm{V} \oplus{ }^{n} \mathrm{H}$, by Proposition 1.5. Conversely, let $n \geq 1$ and $M \in \mathrm{V} \oplus{ }^{n} \mathrm{H}$. Then $\mathrm{K}_{\mathrm{H}}^{(n)}(M) \in$ $\mathrm{V}$, whence $\mathrm{K}_{\mathrm{H}}^{(n)}(M)$ is a $\mathrm{H}$-solvable monoid and so $\mathrm{K}_{\mathrm{H}}^{(m)}\left(\mathrm{K}_{\mathrm{H}}^{(n)}(M)\right)=\left\langle E\left(\mathrm{~K}_{\mathrm{H}}^{(n)}(M)\right)\right\rangle$, for some $m \in \mathbb{N}$. Since $E\left(\mathrm{~K}_{\mathrm{H}}^{(n)}(M)\right)=E(M)$, we have $\mathrm{K}_{\mathrm{H}}^{(m+n)}(M)=\langle E(M)\rangle$, whence $M$ is $\mathrm{H}$-solvable. On the other hand, as $\langle E(M)\rangle$ is a submonoid of $\mathrm{K}_{\mathrm{H}}^{(n)}(M)$, we have that $\langle E(M)\rangle \in \mathrm{V}$. Hence $M \in \mathrm{EV}_{\mathrm{Hsol}}$, as required.

Observe that the equality (4) implies, in some sense independently from Theorem 2.5, that $\mathrm{EV}_{\mathrm{Hsol}}$ is a pseudovariety of monoids. However, notice that Theorem 2.5 is more general.

As a particular instance of (4), we have

$$
\mathrm{Ecom}_{\mathrm{Hsol}}=\mathrm{ESI}_{\mathrm{Hsol}}=\cup_{n \geq 1} \mathrm{SI} m^{n} \mathrm{H} .
$$


By the above discussion on the relation between the operators "Mal'cev product" and "semidirect product", and since the Mal'cev product of pseudovarieties is not associative, we do not see any reason to believe that the equality $\mathrm{V} *^{n} \mathrm{H}=\mathrm{V} m^{n} \mathrm{H}$ holds in general, even when $\mathrm{V} \neq \mathrm{I}$ is a local pseudovariety. The search for examples seems natural and we leave here the question:

Question 4.1 Give examples of pseudovarieties $\mathrm{V}$ of monoids and $\mathrm{H}$ of groups for which $\mathrm{V} *^{n} \mathrm{H}=\mathrm{V} m^{n} \mathrm{H}$ holds.

Since the semidirect product of pseudovarieties is associative, we can state the following proposition which says in particular that the semidirect product of a $\mathrm{H}$-solvable monoid by a $\mathrm{H}$-solvable group is a $\mathrm{H}$-solvable monoid.

Proposition 4.2 One has

$$
\mathrm{V} * \mathrm{G}_{\mathrm{Hsol}}=\mathrm{V} * \cup_{n \geq 1} \mathrm{H}^{n}=\cup_{n \geq 1} \mathrm{~V} *^{n} \mathrm{H} \subseteq \cup_{n \geq 1} \mathrm{~V} m{ }^{n} \mathrm{H}=\mathrm{EV}_{\mathrm{Hsol}},
$$

for any pseudovariety $\mathrm{V}$ of $\mathrm{H}$-solvable monoids.

Let $\mathrm{V} \subseteq \mathrm{DA}$ be a pseudovariety of monoids. It is a $\mathrm{H}$-solvable pseudovariety, by Theorem 2.14. By Proposition 4.2, we have

$$
\mathrm{V} * \mathrm{G}_{\mathrm{Hsol}} \subseteq \mathrm{EV}_{\mathrm{Hsol}}
$$

and we may ask:

Question 4.3 Give examples of pseudovarieties $\mathrm{V}$ of monoids and $\mathrm{H}$ of groups for which the inclusion (5) is in fact an equality.

Related results may be found in [5, 24]. Of course, examples answering positively Question 4.1 would also answer positively the last question.

By Corollary 2.15, we have

$$
\mathrm{EV}_{\mathrm{Hsol}} \subseteq \overline{\mathrm{G}}_{\mathrm{Hsol}} \cap \mathrm{EV}
$$

and one more question may be asked:

Question 4.4 Give examples of pseudovarieties $\mathrm{V}$ of monoids and $\mathrm{H}$ of groups for which the inclusion (6) is in fact an equality.

Attempting to find examples to answer Questions 4.3 and 4.4, the obvious candidates to begin with are $\mathrm{V}=\mathrm{SI}$ and $\mathrm{H}=\mathrm{Ab}$. It follows from Section 3 that they serve as example for Question 4.4. From an example of a semigroup given in [17], it follows that there is an aperiodic monoid whose idempotents commute which does not lie in $\mathrm{SI} m \mathrm{G}_{\text {sol }}$. Since $\mathrm{SI}$ is local, it follows that our candidates are not examples for Question 4.3.

Questions making sense for solvable groups also make sense for solvable monoids. For instance, one can define derived length of a solvable group $G$ : the least integer $n$ such that $G^{(n)}=1$. One can define similarly Abelian kernel length of a finite monoid $M$ as the least integer $n$ such that $\mathrm{K}_{\mathrm{Ab}}^{(n)}(M)=\langle E(M)\rangle$. 
Question 4.5 Given a finite Ab-solvable monoid, compute its Abelian kernel length.

The answer is not "the maximum of the derived lengths of the subgroups of the monoid". In fact, there are aperiodic inverse monoids with derived length $n$, for any positive integer $n$. For instance, the family $\left\{\mathcal{P O} \mathcal{I}_{n} \mid n \in \mathbb{N}\right\}$ provides us such an example (see [13]).

Given a finite monoid $M$ and a pseudovariety $\mathrm{K}$ of groups, one can, by analogy with the definition of group complexity, define the K-complexity of $M$ as the least positive integer $n$ such that

$$
M \in(\mathrm{A} * \mathrm{~K})^{n} * \mathrm{~A} .
$$

holds, if such an integer exists, and $\infty$ otherwise. Note that, for example, a solvable group of derived length $n$ has Ab-complexity at most $n$. Observe that if $\mathrm{K}$ is closed for semidirect products (for instance, if $\mathrm{K}=\mathrm{G}_{\mathrm{Hsol}}$ ) and all subgroups of $M$ belong to $\mathrm{K}$, it follows from Krohn-Rhodes decomposition theorem (see $[1,19]$ ) that there exists an integer $n$ satisfying (7).

The following question may be asked:

Question 4.6 What can be said about the $\mathrm{G}_{\mathrm{Hsol}}$-complexity of a finite $\mathrm{H}$-solvable monoid $M$ ?

Since a monoid consisting of idempotents is aperiodic, i.e. $B \subseteq A$, if it was the case that $\mathrm{V}=\mathrm{B}$ and $\mathrm{H}=\mathrm{Ab}$ were examples for Question 4.3, then the monoids in $\mathrm{EB}_{\text {sol }}$ would

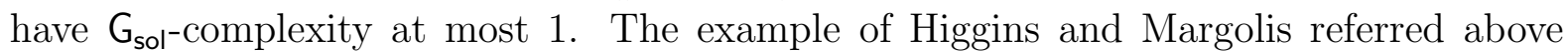
assures us that there are solvable monoids with commuting idempotents outside $\mathrm{SI} * \mathrm{G}_{\text {sol }}$ but this does not give us the guarantee that there do not exist solvable monoids with commuting idempotents with $\mathrm{G}_{\text {sol }}$-complexity greater that 1 .

\section{Acknowledgments}

We wish to thank Jorge Almeida and Benjamin Steinberg for many helpful discussions and comments. We are also acknowledged to the anonymous referee for his/her valuable suggestions.

\section{References}

[1] J. Almeida, Finite Semigroups and Universal Algebra, World Scientific, Singapore, 1995. English translation.

[2] - Finite semigroups: an introduction to a unified theory of pseudovarieties, In G. M. S. Gomes, J.-E. Pin and P. V. Silva (eds.), Semigroups, Algorithms, Automata and Languages, World Scientific, Singapore, 2002, 3-64.

[3] J. Almeida and M. Delgado, Sur certains systèmes d'équations avec contraintes dans un groupe libre, Portugal. Math. 56 (1999) 409-417.

[4] - Sur certains systèmes d'équations avec contraintes dans un groupe libreaddenda, Portugal. Math. 58 (2001) 379-387. 
[5] J. Almeida and A. Escada, On the equation $V * G=E V$, J. Pure and Appl. Algebra 166 (2002) 1-28.

[6] J. Almeida and B. Steinberg, On the decidability of iterated semidirect products and applications to complexity, Proc. London Math. Soc. 80 (2000) 50-74.

[7] C.J. Ash, Inevitable graphs: a proof of the type II conjecture and some related decision procedures, Int. J. Algebra Comput. 1 (1991) 127-146.

[8] D. Barrington and H. Straubing, Superlinear lower bounds for bounded-width branching programs, J. Comp. and System Sci. 50 (1995) 374-381.

[9] M. Delgado, Abelian pointlikes of a monoid, Semigroup Forum 56 (1998) 127-146.

[10] - Commutative images of rational languages and the Abelian kernel of a monoid, Theoretical Informatics and Applications, 35 (2001) 419-435.

[11] - On the hyperdecidability of pseudovarieties of groups, Int. J. Algebra Comput. 11 (2001) 753-771.

[12] M. Delgado and V.H. Fernandes, Abelian kernels of some monoids of injective partial transformations and an application, Semigroup Forum 61 (2000) 435-452.

[13] - Abelian kernels, solvable monoids and the abelian kernel length of a finite monoid, to appear in the proceedings of the workshop "Semigroups and Languages" (Lisboa, November 2002).

[14] V.H. Fernandes, The monoid of all injective order preserving partial transformations on a finite chain, Semigroup Forum 62 (2001) 178-204.

[15] K. Henckell, S. Margolis, J.-E. Pin, and J. Rhodes, Ash's type II theorem, profinite topology and Malcev products. Part I, Int. J. Algebra Comput. 1 (1991) 411-436.

[16] B. Herwig and D. Lascar, Extending partial automorphisms and the profinite topology on free groups, Trans. Amer. Math. Soc. 352 (2000) 1985-2021.

[17] P.M. Higgins and S.W. Margolis, Finite aperiodic semigroups with commuting idempotents and generalizations, Israel J. Math. 116 (2000) 367-380.

[18] J.M. Howie, Fundamentals of Semigroup Theory, Oxford University Press, 1995.

[19] S. Margolis, M. Sapir, and P. Weil, Irreducibility of certain pseudovarieties, Comm. in Algebra 26 (1998) 779-792.

[20] J.-E. Pin, Varieties of Formal Languages, Plenum, London, 1986. English translation.

[21] J. Rhodes, Kernel systems - a global study of homomorphisms on finite semigroups, J. Algebra 49 (1977) 1-45.

[22] L. Ribes and P.A. Zalesskiı̌, On the profinite topology on a free group, Bull. London Math. Soc. 25 (1993) 37-43.

[23] D. J. S. Robinson, "A Course in the Theory of groups", Springer Verlag, 1982.

[24] B. Steinberg, Finite state automata: a geometric approach, Trans. Amer. Math. Soc. 353 (2001) 3409-3464. 
[25] H. Straubing, Families of recognizable sets corresponding to certain varieties of finite monoids, J. Pure and Applied Algebra 15 (1979) 305-318.

[26] M. Suzuki, "Group Theory", vol. I, Springer Verlag, 1980.

[27] The GAP Group, GAP - Groups, Algorithms, and Programming, Version 4.1, Aachen, St Andrews, 1999. (http://www-gap.dcs.st-and.ac.uk/〜gap).

[28] B. Tilson, Categories as algebra: an essential ingredient in the theory of monoids, J. Pure and Applied Algebra 48 (1987) 83-198.

Version of January 31, 2004

Manuel Delgado

Centro de Matemática

Universidade do Porto

Rua do Campo Alegre, 687

4169-007 Porto

Portugal

e-mail: mdelgado@fc.up.pt
Vítor H. Fernandes

Departamento de Matemática

Faculdade de Ciências e Tecnologia

Universidade Nova de Lisboa

Monte de Caparica

2829-516 Caparica

Portugal

$\&$

C.A.U.L.

Av. Prof. Gama Pinto, 2

1649-003 Lisboa

Portugal

e-mail: vhf@fct.unl.pt 